\title{
Leiomyosarcoma Metastatic to the Brain - Clinical Features and Therapeutic Challenges of a Rare Entity
}

\author{
Holger Schlag ${ }^{1 *}$, Jonathan Neuhoff ${ }^{1}$, Christoph Hoffmann ${ }^{1}$, Samiul Muquit ${ }^{2}$ and Frank \\ Kandziora ${ }^{1}$
}

${ }^{1}$ Centre for Spinal Surgery and Neurotraumatology, BG Unfallklinik Frankfurt am Main, Germany

${ }^{2}$ Southwest Neurosurgery Centre, University Hospitals Plymouth NHS Trust, United Kingdom

\begin{abstract}
Brain metastases from leiomyosarcoma are very rare. We present 2 cases of intracranial metastatic leiomyosarcoma, discussing their clinical details, radiological and histological features and treatment outcome. Metastatic leiomyosarcoma to the brain, however, represents a therapeutic challenge, as these tumors are generally resistant to radiation therapy and systemic chemotherapy. Surgery was the treatment of choice in both cases.
\end{abstract}

Keywords

Soft tissue sarcoma, Leiomyosarcoma, Brain metastasis

\section{Introduction}

Soft tissue sarcomas are relatively rare malignant tumors and the reported international incidence rate range from 1.8 to 5 per 100.000 per year [1]. Leiomyosarcomas are one of the most common subtypes, comprising $18 \%$ of all soft tissue sarcomas. The most common sites of occurrence are retroperitoneal, uterus and major vessels [2]. However, leiomyosarcomas of the Central Nervous System have only been reported sporadically and are a rare cause of brain metastasis in soft tissue cancer [3]. The incidence is thought to be higher in immunocompromised patients; An association with AIDS and Ebstein-Barr Virus (EBV) has been suggested [4-6]. Both primary cerebral leiomyosarcomas [7] and cerebral metastases [8] have been reported but therapeutic challenges remain. We present two cases of intracranial metastatic leiomyosarcoma and discuss treatment options, our management and outcomes.

\section{Case Description}

Case 1: A 68-years-old male patient presented with a 2 weeks history of progressive left arm weakness. Past medical history was unremarkable. There was no history of smoking, IV drug abuse, or suggestion of acquired immunodeficiency. Physical examination was only remarkable for a left arm paresis. There were no other neurological deficits. A complete blood count and blood chemistry were within the normal ranges. Viral serology was negative for HIV and Epstein Barr Virus (EBV). CT of the brain revealed multiloculated supraand infratentorial lesions and no hydrocephalus.
Additional Magnetic resonance imaging (MRI) showed in homogeneously enhancing lesions, which exhibited an increased signal in T2 and flair sequences. Most notable were lesions in the right cerebellar hemisphere, in the right precentral gyrus and in the left posterior horn of the left lateral ventricle, in addition two smaller lesions scattered in both cerebral hemispheres. The largest (right precentral) lesion was associated with local mass effect and perilesional oedema. At this stage in his treatment the etiology of these tumors was unknown (Figure 1).

The patient decided to undergo surgical resection of this lesion, which we performed awake. Intra-operatively we found a poorly vascularized tumor with good demarcation. A complete macroscopic resection was achieved with satisfactory cortical- subcortical stimulation and neurological

*Corresponding author: Dr. Holger Schlag, Centre for Spinal Surgery and Neurotraumatology, BG Unfallklinik Frankfurt am Main gGmbH, Friedberger Landstraße 430, D-60389 Frankfurt am Main, Germany, Tel: + 49-(0)69/475-2020, Fax: +49(0)69/475-2018

Accepted: July 03, 2021

Published online: July 05, 2021

Citation: Schlag H, Neuhoff J, Hoffmann C, et al. (2021) Leiomyosarcoma Metastatic to the Brain - Clinical Features and Therapeutic Challenges of a Rare Entity. J Clin Surg Pathol $3(1): 21-26$ 


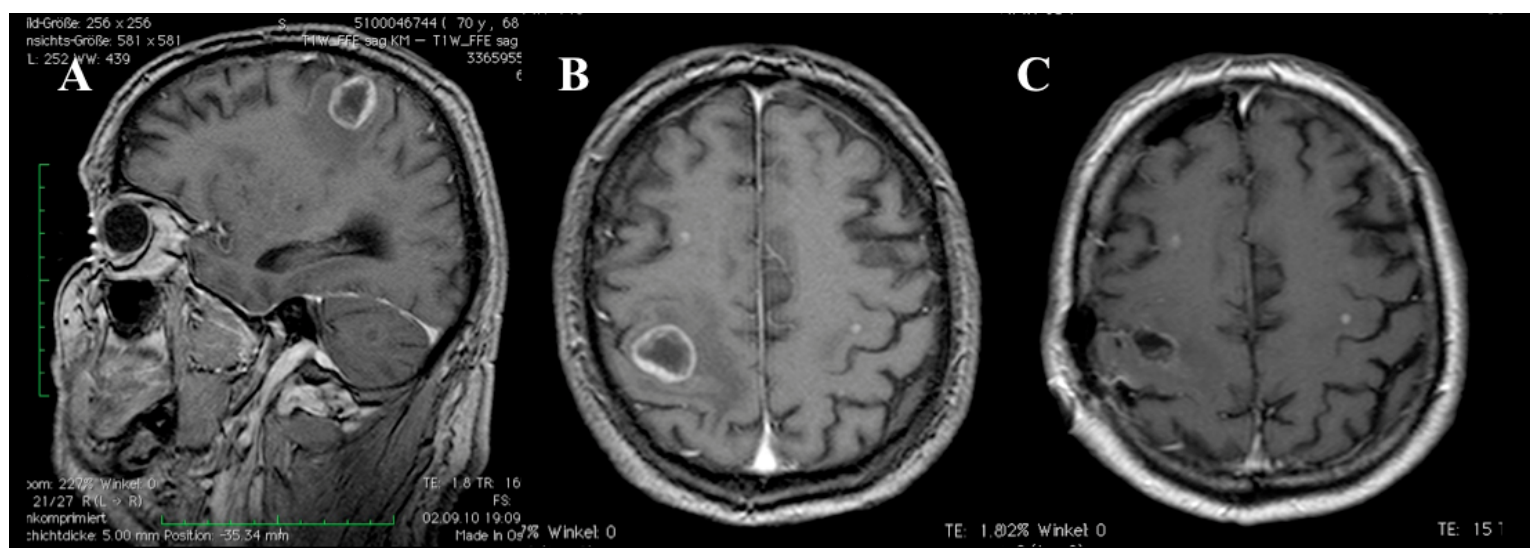

Figure 1: Magnetic resonance images (MRI) after gadolinium administration obtained from Patient 1. A) Preoperative sagittale; B) Axial T1-weighted images revealing a right central non-homogenic tumor; C) Postoperative axial T1-weighted image showed a complete tumor resection.

examination during surgery. Post-surgery the patient had no change from the pre-operative neurological deficit. MRI confirmed total resection of the right precentral lesion.

Histological examination of the lesion revealed a well differentiated, pleomorphic tumor composed of intersecting bundles of eosinophilic spindle cells with round, oval to elongated, hypercromatic nuclei. An increased mitotic rate with atypical mitotic figures and focal necrosis were identified. Immunohistochemistry showed immune-reactivity for smooth-muscle-actin (SMA) and vimentin. Some tumor cells showed a weak staining for muscle-specific-actin (HHF35). The tumor cells were negative for S100, desmin, myoD1 and CD34. MIB-1 index was 50\%. Thus, histological and immune-histochemical examinations were consistent with leiomyosarcoma (Figure 2). Following the histopathological finding a CT scan of the chest, abdomen and pelvis as well as $\mathrm{MRI}$ of the spine was performed to find the primary lesion and establish stage of disease. The CT scan of the chest showed a small lesion of $8 \mathrm{~mm}$ diameter in the right middle lobe of the lung with enlarged right hilar lymph nodes. CT abdomen demonstrated a small, $14 \mathrm{~mm}$, hypodense subcapsular splenic lesion. Both of these lesions were interpreted as suspicious for metastatic foci. Due to the unclear origin of the leiomyosarcoma, additional tests were performed. However endoscopy, colonoscopy and abdominal ultrasound couldn't reveal any primary tumor. The patient was unstable and died after 26 days following pulmonary infection.

Case 2: A 76-years-old male, with a previous history of pleural leiomyosarcoma with secondary infiltration of the upper lobe of the right lung and right $3^{\text {rd }}$ and $4^{\text {th }}$ intercostal ribs and muscles, presented with new onset left facial and left arm myoclonic seizures with no secondary generalized seizures. The patient developed left hemiplegia, which persisted postictaly but there were no other additional notable physical signs. Blood tests showed chronic microcytic anaemia with no other significant abnormalities. Viral serology was negative for HIV and EBV. CT revealed a right parietal lesion.

MRI T1 sequence of the Brain with contrast showed heterogenously enhancing right parietal durally based intracranial lesion casuing significant mass effect (Figure 3).
The lesion showed increased signal in $\mathrm{T} 2$ and flair sequences, raising a possible diagnosis of meningioma. The patient agreed to have surgery and underwent a right parietal craniotomy. Intra-operatively we found a well demarcated and poor vascularized tumor, which was attached to the dura. Total macroscopic resection was achieved.

In the Postoperative course the patient showed a marked improvement of the left sided weakness, and only the left facial weakness persisted. Post-operative contrast enhanced CT of the head demonstrated total tumor resection.

The histologic examination demonstrated a pleomorphic tumour, composed of intersecting bundles of eosinophilic spindle cells with round-oval to elongated, hypercromatic nuclei and single mitotic figures. The tumour cells showed immunreactivity for smooth-muscle-actin (SMA) and vimentin. The tumour cells were negative for S100, CD 34, desmin and pa-cytokeratin MNF 116. MIB-1 index was $40 \%$. Therefore, histology and immunohistochemistry confirmed the diagnoses of metastatic leiomyosarcoma (Figure 4).

Although the patient was counseled thoroughly about adjuvant therapy, he declined further treatment and follow up.

\section{Discussion}

Soft tissue sarcomas are relatively rare tumors overall [1]. Leiomyosarcoma make up a large proportion of these tumors and are known to originate from the uterus, gastrointestinal tract, skin and blood vessels. Primary intracranial leiomyosarcoma and metastatic cerebral spread from other primary sites are very rare [2]. The incidence is unknown, and only a limited number of cases have been reported in the literature [3]. Primary intracranial leiomyosarcoma shows preponderance in immune compromised patients, suggesting an association between the neoplasm and AIDS as well as EBV $[5,6,9,10]$. In these cases the tendency is for tumors to develop in the skull base, particularly the sellar region $[9,11,12]$.

Both of the patients we present here had metastatic leiomyosarcoma. Espat NJ, et al., [2]. studied 3829 patients with soft tissue sarcoma. In this study a prevalence of brain metastasis was found to be less than $1 \% .8$ patients out of 
Citation: Schlag H, Neuhoff J, Hoffmann C, et al. (2021) Leiomyosarcoma Metastatic to the Brain - Clinical Features and Therapeutic Challenges of a Rare Entity. J Clin Surg Pathol 3(1):21-26

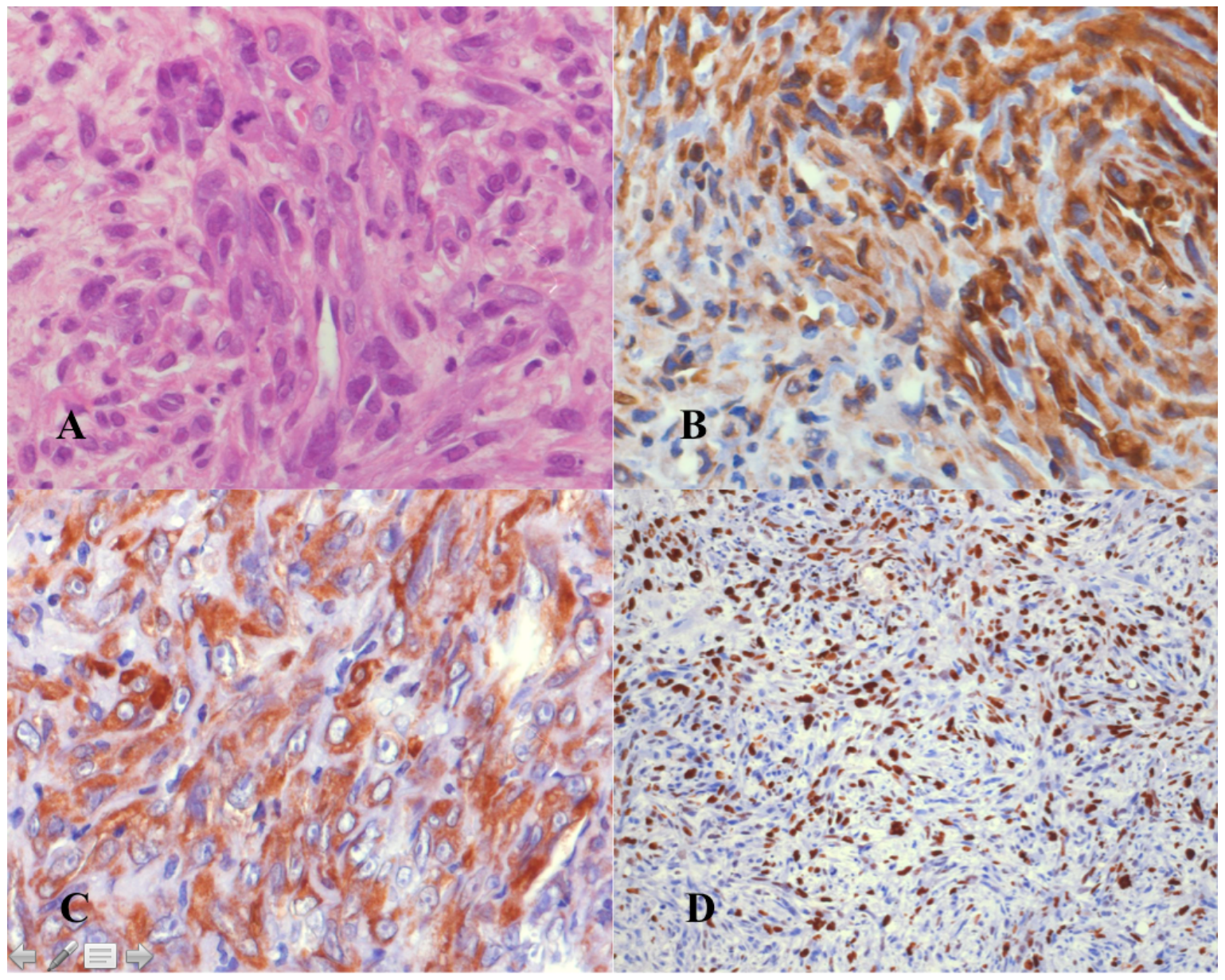

Figure 2: Histopathological features of tissue samples obtained during operation of Patient 1. A) Pleomorpic, spindle sharped tumor cells with round-oval to elongated nuclei and some prominent nucleoli and one single mitotic figure. H\&E staining, original magnification: 400x; B) Tumor cells express vimentin. Immunhistochemistry with anti-Vimentin counterstained with hemalum, original magnification: 400x; C) Tumor cells positive for smooth-muscle-Actin (SMA). Immunohistochemistry with anti-Actin (SMA) counterstained with hemalum, original magnification: 400x; D) MIB-1 index was 50\%. Immunohistochemistry with anti-Ki67 (MIB-1) counterstained with hemalum, original magnification: 200x.

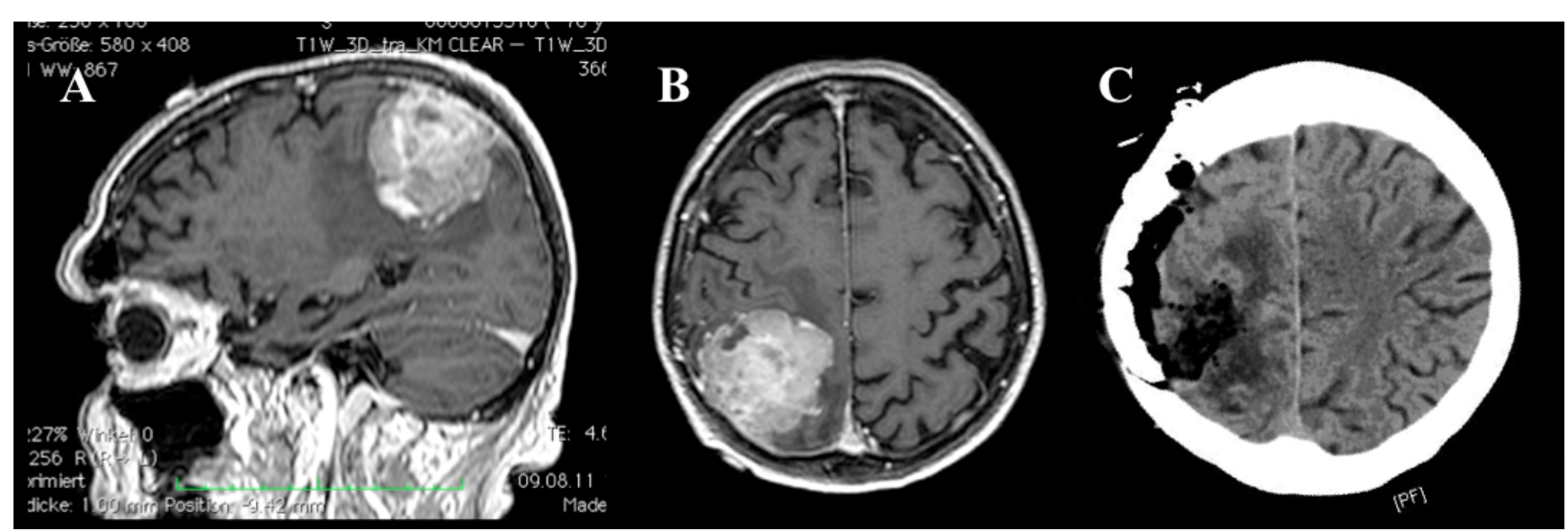

Figure 3: Magnetic resonance images after gadolinium administration obtained from Patient 2. A) Preoperative sagittale; B) Axial T1weighted images revealing a right central tumor; C) Postoperative CT showed a complete tumor resection. 


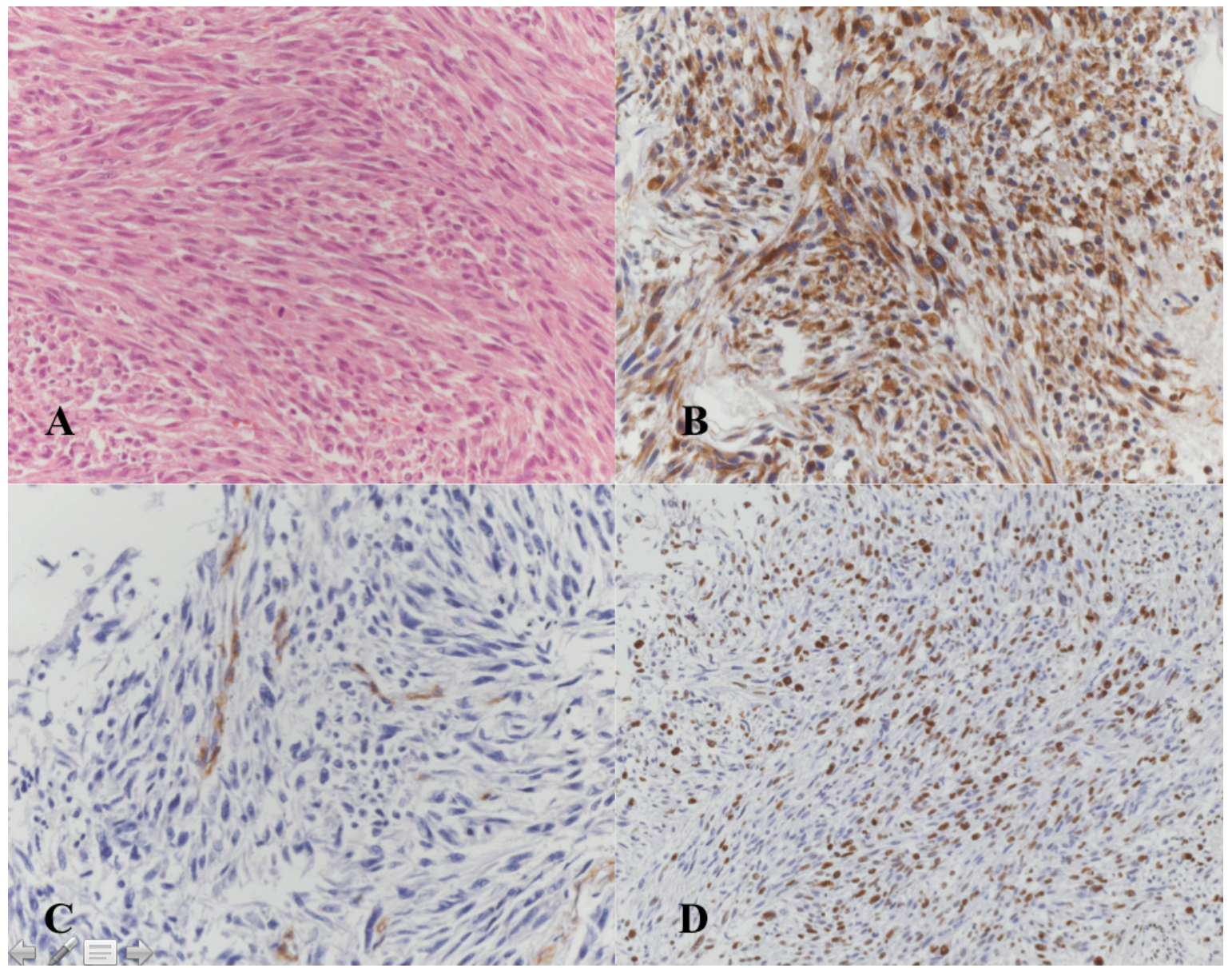

Figure 4: Histopathological features of tissue samples obtained during operation of Patient 2. A) Pleomorphic tumor composed of intersecting bundles of eosinophilic spindle cells with round-oval to elongated, hypercromatic nuclei and one single mitotic figure. $\mathrm{H} \& \mathrm{E}$ staining, original magnification: 200x; B) Tumor cells express vimentin. Immunohistochemistry with anti-Vimentin counterstained with hemalum, original magnification: 200x. C) Single tumor cells positives for smooth-muscle-Actin (SMA). Immunohistochemistry with antiActin (SMA) counterstained with hemalum, original magnification: 200x. D) MIB-1 index was 40\%. Immunohistochemistry with anti-Ki67 (MIB-1) counterstained with hemalum, original magnification: 100x.

40 showed brain metastasis leiomyosarcoma. This study showed that the most frequent soft tissue sarcoma subtype with metastatic brain involvement was leiomyosarcoma [2]. This pathology represents a therapeutic challenge as little is known of treatment outcomes. In addition, leiomyosarcoma tends to exhibit hematogenous spread to the lung prior to the appearance of brain metastases, which has an impact on survival $[2,13]$. Both of our cases showed pulmonary tissue involvement on staging $\mathrm{CT}$.

There are no unique features on imaging which can establish the diagnosis of intracranial leiomyosarcoma. Past medical history, clinical suspicious and histological examinations are necessary. The diagnosis of leiomyosarcoma is confirmed using histological features of smooth muscle cells and immunohistochemistry. The tumor cells are elongated with tapering cytoplasmic processes, convoluted nuclei and basement membrane material around the cytoplasmic membrane. Immunohistochemical testing excluded other differential diagnoses, which include high-grade astrocytoma, malignant fibrous histocytoma, and meningioma. Leiomyosarcoma is characteristically positive for smooth muscle actin and cytokeratin staining and negative for S-100 protein and epithelial membrane antigen.

Both of our patients were treated with complete resection of the Tumor with safe margin. Intraoperative monitoring was used in both cases to minimize the risk of worsening neurological function. Due to the cardiopulmonary instability of the first patient and the refusal of the second patient both patients didn't receive any further therapy. However, as these cerebral metastasis from leiomyosarcoma is very rare the evidence showing a survival improvement with adjuvant chemo- or radiotherapy is lacking. To the best of our knowledge there are no published studies directly examining effects of cranial radiotherapy on survival. The use of radiation therapy in the adjuvant setting does not appear to be beneficial with extracranial leiomyosarcomas. Published evidence indicates no benefit in overall survival but local recurrence rate may be reduced [14-16]. If these results are extrapolated to cranial disease it implies no benefit from cranial radiotherapy. The use of adjuvent chemotherapy is controversial. Treatment with doxorubicin or with the combination of gemcitabine-docetaxel in cases 
Citation: Schlag H, Neuhoff J, Hoffmann C, et al. (2021) Leiomyosarcoma Metastatic to the Brain - Clinical Features and Therapeutic Challenges of a Rare Entity. J Clin Surg Pathol 3(1):21-26

of uterine leiomyosarcoma had no effect on recurrence rates or overall survival [17]. The same conclusions were reached with treatment with doxorubicin-ifosfamide [18]. However, a beneficial effect on overall survival was found, but only in high-grade (grade 3) histology, where survival improved from $45 \%$ to $58 \%$ [19].

Gamma Knife radiosurgery (GKRS) has been tried on cranial metastases from leiomyosarcoma. Shepard, et al., [20] report use of GKRS in 1 patient. However Karnofsky Performane status in this patient prior to GKRS was 20 and the patient died within one month of treatment due to progression of primary disease [20]. Yamada, et al. also presented a case report on a patient with multiple cerebral leiomyosarcoma metastases treated with surgery and GKRS to the residual tumors. However followup after GKRS was only 6 months as the patient subsequently died from intraabdominal haemorrhage [21]. At 6 months there was no evidence of progression. Flannery, et al. treated 4 patients and suggest a good clinical response. Survival following GKRS ranged from 2.5 to 30 months. Two of their patients were alive at the end of their study, 1 died of progressive systemic disease and the remaining patient died of an unknown cause. Their conclusion from GKRS treatment of a total of 21 patients with brain metastases from a variety of soft tissue sarcomas was, that local tumour control rates were higher in patients with tumours measuring $<0.5 \mathrm{~cm}^{3}$. Nevertheless further evidence of the efficacy of GKRS is necessary before its use in these cases can be widely advocated [22].

As such current treatment for cranial leiomyosarcoma (primary or metastatic), as with other sarcomas, rests primarily with complete surgical resection $[23,24]$. Surgical resection of sarcoma metastases to the brain, overall, is associated with a relatively low risk of operative death and results in improvement in neurological function with patients with systemic controlled of their primary disease and certain histological subtypes [24].

\section{Conclusion}

Treatment of cranial leiomyosarcoma, either primary or metastatic, remains a therapeutic challenge. The resistance to radiotherapy and the current uncertainties on the effectiveness of chemotherapy and GKRS mean that surgical resection remains the primary, if not the only treatment option. In our two cases we achieved total surgical resection, both macroscopically and radiographically, without worsening of neurological function. As with other cranial sarcoma metastases complete surgical resection can be achieved safely and offers the patient the best chance of survival.

\section{Financial Support and Sponsorship}

Nil.

\section{Conflict of Interest}

There are no conflicts of interest.

\section{References}

1. Wibmer C, Leithner A, Zielonke N, et al. (2010) Increasing incidence rates of soft tissue sarcomas? A population-based epidemiologic study and literature review. Ann Oncol 21: 1106-1111.
2. Espat NJ, Bilsky M, Lewis JJ, et al. (2002) Soft tissue sarcoma brain metastases. Prevalence in a cohort of 3829 patients. Cancer 94: 2706-2711.

3. Shweikeh F, Bukavina L, Saeed K, et al. (2014) Brain metastasis in bone and soft tissue cancers: A review of incidence, interventions, and outcomes. Sarcoma 2014: 475175.

4. Blumenthal DT, Raizer JJ, Rosenblum MK, et al. (1999) Primary intracranial neoplasms in patients with HIV. Neurology 52: 1648-1651.

5. Brown HG, Burger PC, Olivi A, et al. (1999) Intracranial leiomyosarcoma in a patient with AIDS. Neuroradiology 41: 35-39.

6. Gupta S, Havens PL, Southern JF, et al. (2010) Epstein-Barr virus-associated intracranial leiomyosarcoma in an HIV-positive adolescent. J Pediatr Hematol Oncol 32: e144-e147.

7. Aeddula NR, Pathireddy S, Samaha T, et al. (2011) Primary intracranial leiomyosarcoma in an immunocompetent adult. J Clin Oncol 29: e407-e410.

8. Gautschi OP, Hottinger AF, Lobrinus JA, et al. (2014) Isolated cerebral metastasis of a triceps muscle leiomyosarcoma: A case report. British Journal of Neurosurgery 28: 400-402.

9. Anderson WR, Cameron JD, Tsai SH (1980) Primary intracranial leiomyosarcoma. Case report with ultrastructural study. J Neurosurg 53: 401-405.

10. Citow JS, Kranzler L (2000) Multicentric intracranial smoothmuscle tumor in a woman with human immunodeficiency virus. Case report. J Neurosurg 93: 701-703.

11. Kleinschmidt-DeMasters BK, Mierau GW, Sze Cl, et al. (1998) Unusual dural and skull-based mesenchymal neoplasms: A report of four cases. Hum Pathol 29: 240-245.

12. Kroe DJ, Hudgins WR, Simmons JC, et al. (1968) Primary intrasellar leiomyoma. Case report. J Neurosurg 29: 189-191.

13. Haykal HA, Wang AM, Zamani A (1987) Leiomyosarcoma metastatic to the brain: CT features and review. AJNR Am J Neuroradiol 8: 911-912.

14. Mahdavi A, Monk BJ, Ragazzo J, et al. (2009) Pelvic radiation improves local control after hysterectomy for uterine leiomyosarcoma: A 20-year experience. Int J Gynecol Cancer 19: 1080-1084.

15. Reed NS, Mangioni C, Malmstrom H, et al. (2008) Phase III randomised study to evaluate the role of adjuvant pelvic radiotherapy in the treatment of uterine sarcomas stages I and II: An European Organisation for Research and Treatment of Cancer Gynaecological Cancer Group Study (protocol 55874). Eur J Cancer 44: 808-818.

16. Sampath S, Schultheiss TE, Ryu JK, et al. (2010) The role of adjuvant radiation in uterine sarcomas. Int J Radiat Oncol Biol Phys 76: 728-734.

17. Omura GA, Blessing JA, Major F, et al. (1985) A randomized clinical trial of adjuvant adriamycin in uterine sarcomas: $A$ Gynecologic Oncology Group Study. J Clin Oncol 3: 1240-1245.

18. Woll PJ, Reichardt P, Le Cesne A, et al. (2012) Adjuvant chemotherapy with doxorubicin, ifosfamide, and lenograstim for resected soft-tissue sarcoma (EORTC 62931): A multicentre randomised controlled trial. Lancet Oncol 13: 1045-1054.

19. Italiano A, Delva F, Mathoulin-Pelissier S, et al. (2010) Effect of adjuvant chemotherapy on survival in FNCLCC grade 3 soft tissue sarcomas: A multivariate analysis of the French Sarcoma Group Database. Ann Oncol 21: 2436-2441.

20. Shepard MJ, Fezeu F, Lee CC, et al. (2014) Gamma knife radiosurgery for the treatment of gynecologic malignancies metastasizing to the brain: Clinical article. J Neurooncol 120: 515-522. 
21. Yamada S, Yamada SM, Nakaguchi H, et al. (2011) A case of multiple brain metastases of uterine leiomyosarcoma with a literature review. Surg Oncol 20: e127-e131.

22. Flannery T, Kano H, Niranjan A, et al. (2010) Gamma knife radiosurgery as a therapeutic strategy for intracranial sarcomatous metastases. Int J Radiat Oncol Biol Phys 76: 513-519.
23. Bindal RK, Sawaya RE, Leavens ME, et al. (1994) Sarcoma metastatic to the brain: Results of surgical treatment. Neurosurgery 35: 185-190.

24. Fox BD, Patel A, Suki D, et al. (2009) Surgical management of metastatic sarcoma to the brain. J Neurosurg 110: 181-186. 